trois tuyaux consécutifs, on creusa le bélon sur une profondeur d'environ 12 centimètres, on disposa une légère charpente métallique soutenant des faisceaux circulaires formés de fil d'acier à haute résistance, puis on noya complètement ces faisceaux dans une nouvelle application de béton, de façon à obtenir un blindage. On donna à la surface de ce béton la forme cylindrique exacte des luyaux el on reconstitua entièrement ces derniers avec de nouvelles tôles soudées sur place. Cette opération réussit aussi complètement.

Dans la dernière installation de Mese, on déplora aussi un mauvais fonctionnement du système de drainage. Au début, les drainages fonctionnaient très bien, mais à la longue, ils commencèrent à s'obstruer avec des dépôts calcaires laissés par l'eau drainée. Lors d'un vidage de la conduite, exécuté peul-être un peu trop rapidement, les drainages ne permirent pas une évacuation suffisamment rapide de l'eau accumulée entre les tuyaux et le béton et il en résulta.l'écrasement de quelcutes tuyaux. Là encore, on procéda à la réparation avec le système habituel de la réfection partielle des tuyaux avec, de nouvelles tôles. Mais comme 1 s'agissait de tuyaux frettés, on dut prendre des précautions spéciales pour les soudures afin de ne pas altérer les qualilés du fil de frettage venant en conlacl avec les tôles. En fait, tout réussit parfaitement.

Dans les figures 45 à 54 sont représentées quelques phases pariıculières de ces diverses réparations.

I'ai eu l'occasion de m'occuper de toutes ces réparations, sur ces diverses installations, et ce qui m'a le plus frappé, c'est la facilité relative avec laquelle on put réparer ces conduites. Naturellement, cette opération ne doit pas être confiée à qui que ce soit ; toutefois, pendant le cours même de la réparation, on acquiert une première certitude sur la réussite. Le résultat fut d'ailleurs toujours parfait.

Pour conclure, je dirai que le système des conduites élastiques constitue un essai très ingénieux et très intéressant. La somme de ses avantages est considérable et les inconvénients, qui se sont manifestés dans les trois grandes installations construites, sont peu nombreux et tous parfaitement remédiables. Au lieu donc d'exclure ce système, il convient de le reprendre, et mieux encore, de le poursuivre. Il y a de nombreux cas, dans les installations à réaliser, pour lesquels l'adoption des conduites élastiques apporterait une notable simplification du problème de la conduite forcée.

Avec une bonne organisation de chantier et de larges moyens d'action, ce système doit procurer une économie dans le coût de l'installation. Il apportera aussi, s'il est bien exécuté, une amélioration notable du rendement et une économie dans l'exercice.

Il eût été presque inutile de faire mention de ce type de conduite, si l'on n'avait pas pu citer les installations dans lesquelles il fut adopté, les inconvénients constatés, les réparations exécutées. C'est pourquoi, je remercie sincèrement la Société Générale Italienne Edíson d'Électricité, la Société Électrique Interrégionale Cisalpine et l'ingénieur Pietro Marinoni, pour m'avoir aimablement autorisé à citer les expériences des conduites de Pallanzeno, de Rovesca et de Mese, auxquelles j'ai collaboré.

\section{Traduit de l'« Energia Elettrica "par}

Marc Marguerat, Ingénieur.

\title{
Adduction des eaux de la source d'Ain-el-Delbeh pour l'alimentation de la banlieue de Beyrouth (Liban)
}

\author{
par ArIe Visser (Ingénieur Hydraulicien)
}

Beyrouth, ville de près de 200.000 habitants, est le port d'émigration de la Syrie. C'est à Beyrouth que s'embarquent les pèlerins mahométans de toute la Syrie, de Mésopotamie, de Perse et même d'Afghanistan qui vont à La Mecque, en Arabie. La Syrie, cette terre classique, se réveille d'un sommeil de plomb qui a duré pendant des siècles. N'oublions pas que le berceau de l'humanité se trouvait dans ces parages. Le climat y est délicieux au printemps et en automne. Pendant les grandes chaleurs de l'été, des touristes, arrivés de l'Egypte, de Palestine et de la côte Syrienne, viennent passer leurs vacances dans les montagnes à l'est de Beyrouth.

Les charmes multiples du Liban ne sont pas très connus. En arrivant avec le vapeur, le panorama de la ville de Beyrouth se déroule devant le spectateur ravi, car sa beauté n'est pas inférieure à celle de Naples et de son golfe célèbre. Au premier plan, les eaux bleues de la Méditerranée, la baie de Saint-Georges autour de laquelle se groupe Beyrouth, ville de caractère oriental avec ses maisons blanches, ses rues étroites, éclairées par un soleil impitoyable en été, projetant des ombres violettes. On fail le commerce dans les rues, où des fruits de toutes sortes sont entassés, surtout ces belles oranges du Proche-Orient, avec des étals de viande et de volaille, et où les artisans exercent leur métier.
Des contrastes remarquables caractérisent la vie orientale Les automobiles les plus modernes passent auprès de cavaliers montés sur des ânes ; des femmes du monde, et des Bédouines pieds nus, pantalon large, marchant la tète haute, pleine de grâce, se rencontrent dans les rues et sur la grande route de Damas, dont la construction admirable a été terminée dans les dernières années, sous le Gouvernement français.

Les montagnes, avec leurs sommets chauves, possèdent une grande beauté. Les couleurs gris ou rouille de fer s'harmonisent si bien avec un ciel presque toujours bleu sur lequel se dessine à l'arrière-plan, la cime neigeuse du mont Hermon. Il n'est rien de saisissant.comme une vue sur cette montagne du côté du Liban ou de la plaine de Bekaa.

Pendant leur occupation, les Turcs ont exploité le pays d'une façon rigoureuse. Les grandes forêts de cèdres du Liban, mentionnées dans la Bible, ont été abattues. Il faudrait reboiser ces montagnes, comme en Palestine, pour améliorer la précipitation atmosphérique, car il y a un cercle vicieux entre les montagnes chauves, la sécheresse et la stérilité ; cela ajouterait à la gloire du Gouvernement français, qui a déjà fait tant de bien pour ce pays : développé le commerce, organisé la police, créé un réseuu de routes admirables de centaines de kilomètres, etc. 
En Syrie, l'eau c'est la richesse. Où il y a de l'eau, il y a de la culture et du blé, des citrons, des amandes, des bananes avec leurs feuilles gigantesques et les légumes poussent en abondance. Dans ce pays du Liban, la plaine de Békaa et l'Anti-

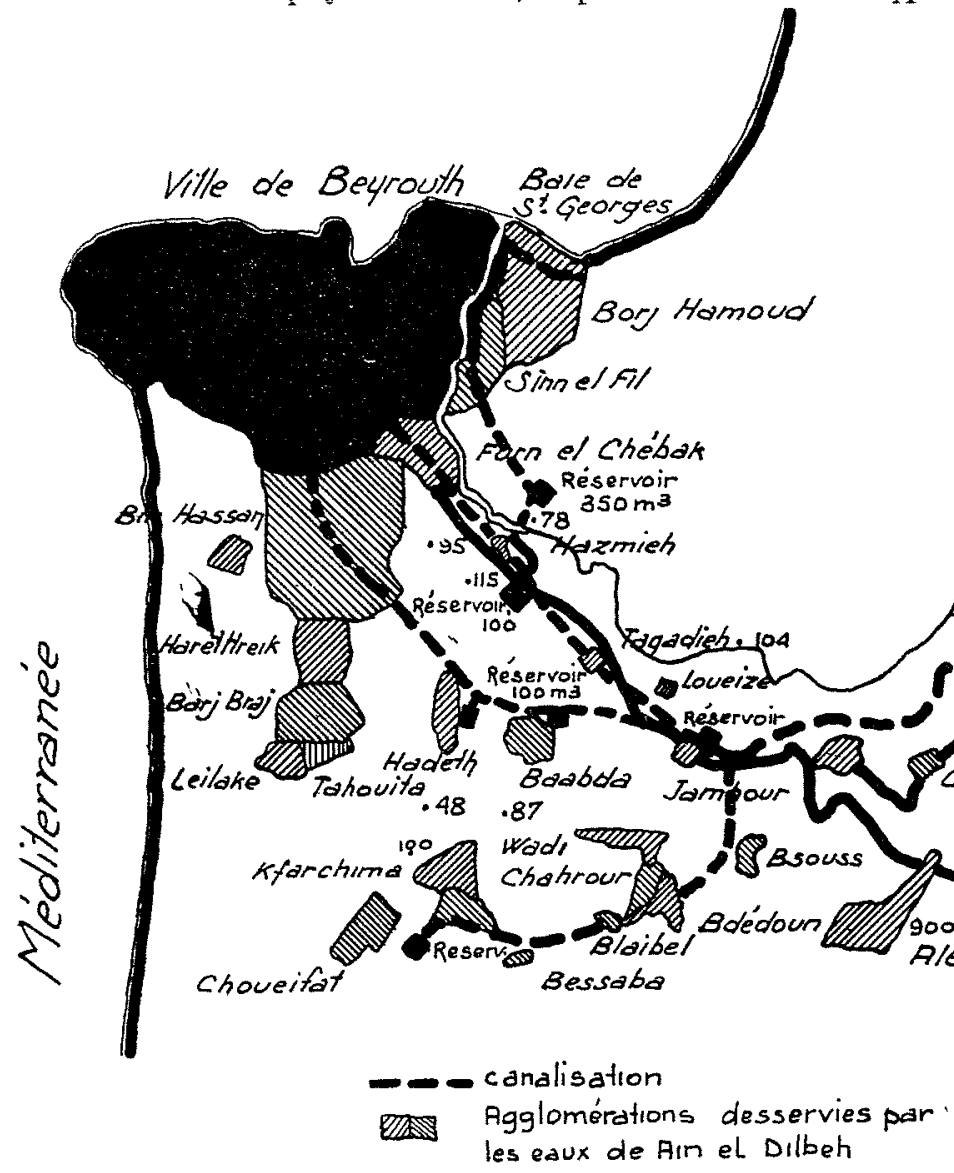

Liban et plus loin, à l'est de Damas, dans le grand désert de Syrie qui s'étend à l'infini vers Bagdad et encore plus à l'est vers la Perse, il y a beaucoup de terre fertile, mais l'eau manque. Dans l'antiquité, pendant le règne des Romains, ce pays a été cultivé. On trouve même, datant de cette période, des traces d'irrigation dans le désert, entre Damas et Bagdad, et même des citernes en Mésopotamie.

La ville de Beyrouth, située au bord de la Méditerranée, est alimentée en eau potable par une station de pompage se trouvant à côté de la rivière au nord de la ville, mais les alentours où se sont développés les centres, ayant à la fois un caractère urbain et rural, en étaient dépourvus. Pour toutes ces agglomérations, dont la population atteint en totalité 42.000 habitants, les captages, les stations de pompage et les canalisations installées par les différents fournisseurs d'eau dans ces localités laissaient à désirer et ne correspondaient plus au développement de la région.

Cet état de choses, datant déjà d'avant guerre, attira l'initiative de certains entrepreneurs du pays qui essayèrent d'obtenir la concession pour l'alimentation de cette vaste région par les eaux d'Ain-el-Delbeh, la seule source dans les environs, dint le débit, la qualité d'eau et la situation topographique permettaient de résoudre le problème. Mais les difficultés d'ordre judiciaire, financier et technique de cette entreprise furent cause de l'insuccès de l'affaire jusqu'à l'année passée. Enfin, en 1933, à Beyrouth, s'organisa la Sociélé Générale d'Entreprises Hydrauliques S. A., qui s'est chargée en premier lieu de l'adduction d'Ain-el-Delbeh. La Société a obtenu du Gouvernement la concession, et cette fois-ci l'entreprise a réussi grâce à la collaboration d'un groupe syrien et d'un groupe hollandais. L'importance pour le Liban et sa capitale, Beyrouth, de ces travaux, effectués d'ailleurs à l'époque de la crise, mérite qu'on en rende compte d'une manière sommaire.

Le schéma du réseau d'adduction est présenté sur la fig 1. La canalisalion principale, de $325 \mathrm{~m} / \mathrm{m}$. de diamètre, va, longeant les vallées des rivières, de la source au réservoir principal de Jamhour, situé sur la crête qui domine toute la zone alimentée. Du réservoir de Jamhour jusqu'aux réservoirs locaux l'eau est amenée par les trois canalisations secondaires :

$1^{0}$ Canalisation de Fayadie-Hazmieh-Sinn el Fil ;

$2^{\circ} \quad-\quad$ de Baabda, Hadeth, Borj, Brayné et

$3^{\circ} \quad-\quad$ Hazmieh, Furn el Chebak-Chiah.

Une quatrième canalisation secondaire, pour les villages de Bsouss, Bdédoun, Wadi Chahrour et ensuile Kfarchima et Choueifat, se montra nécessaire pendant l'exé cution des travaux.

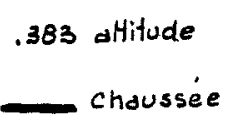

Fig. 1. - Schéma des canalisations d'adduction.

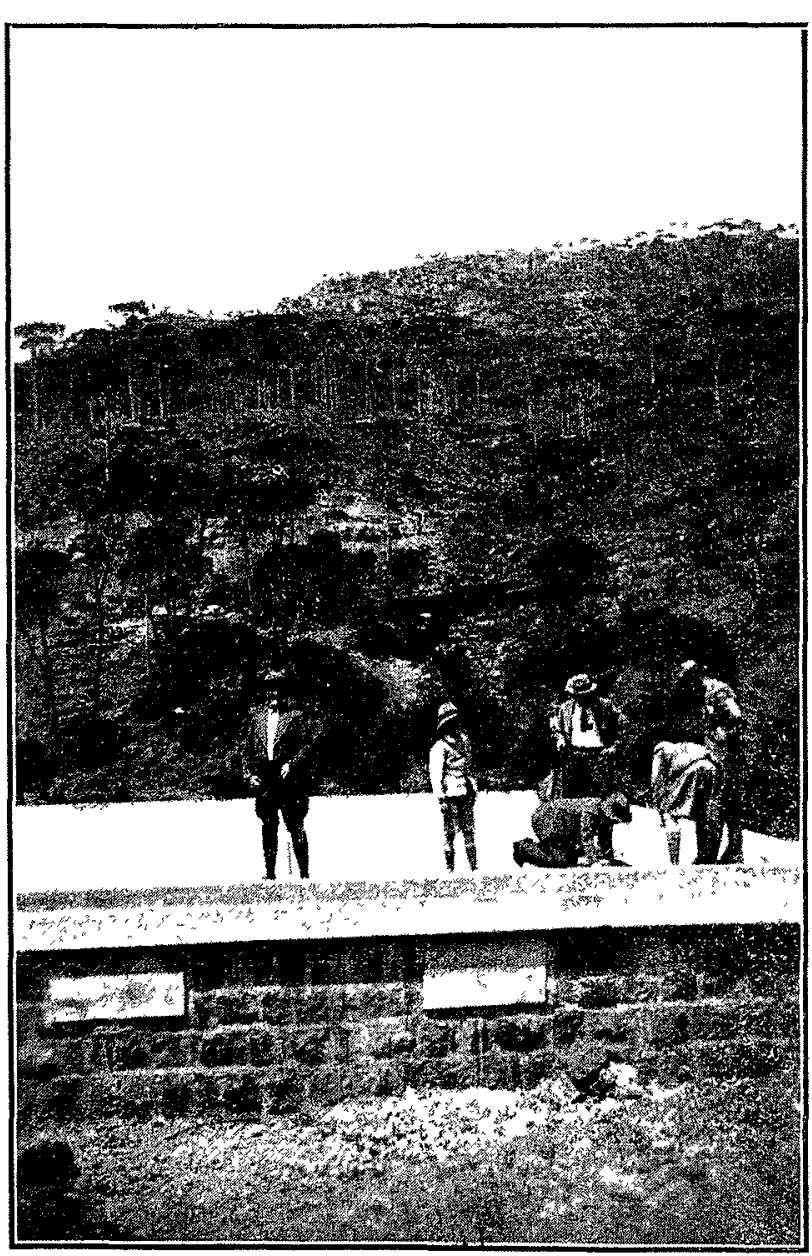

Frg. 2. - Bâtiment de captage: 
Les longueurs des canalisations sonl les suivantes: Canalisation principale de la source jusqu'à Jamhour... secondaire de Jamhour jusqu'au réservoir de Sinn el fill . . . . . . . . . . . . . secondaire de Jamhour jusqu'au réservour

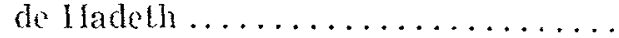
secondaire de Jamhour jusqu'au réservoir de Choteilat ................. secondaire de Jamhour jusqu'au réservoir de llasmieh, lurn el Chebak-Chiah.

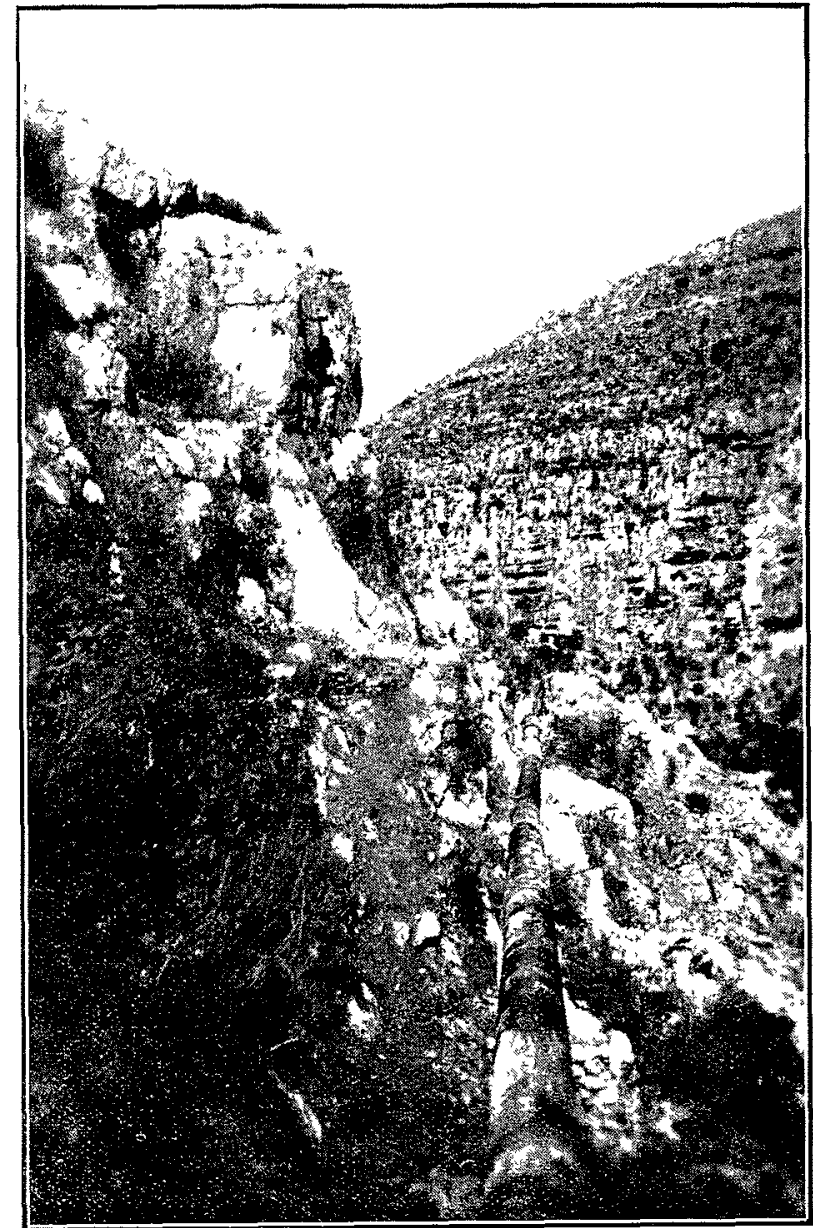

Fıg. 3. - Tronçon difficile de la canalisation. telle quelle. La surface d'émergence, après nettovage, a été $14 \mathrm{~km}$.

$6 \mathrm{~km}$. recouverte par le bâtiment de captage, dont les dimensions en plan dépendaient de la dispositi-n des filets d'eau. Le mur nord

$4,3 \mathrm{~km}$. $6,6 \mathrm{~km}$. $1 \mathrm{~km}$.

Ia construction de ces canalisatıons a élé terminée au mois d'octobre 1934.

Ia source d'Ain-Delbeh, dont le débil a l'époque d'étiage esı de $10.000 \mathrm{~m}^{3}$ par 24 heures, se trouve près du fond même de la profonde vallée de Hammane. Elle émerge à la cote 513 . L.e bassin de réception de la source esl forme par des grosses masses calcaires, constiluant les propres récepleurs des précipitations atmosphériques, qui remplissent d'eau de pluie et de neige leurs innombrables fissures, cavilés el grotles. La règle générale - capter la source sans changer son niveau -.. dul ètre surtout observée dans le cas d'Ain-el-Delbeh, émergeant dans un terrain formé d'un mélange d'argile et de pierres. La source a été captée

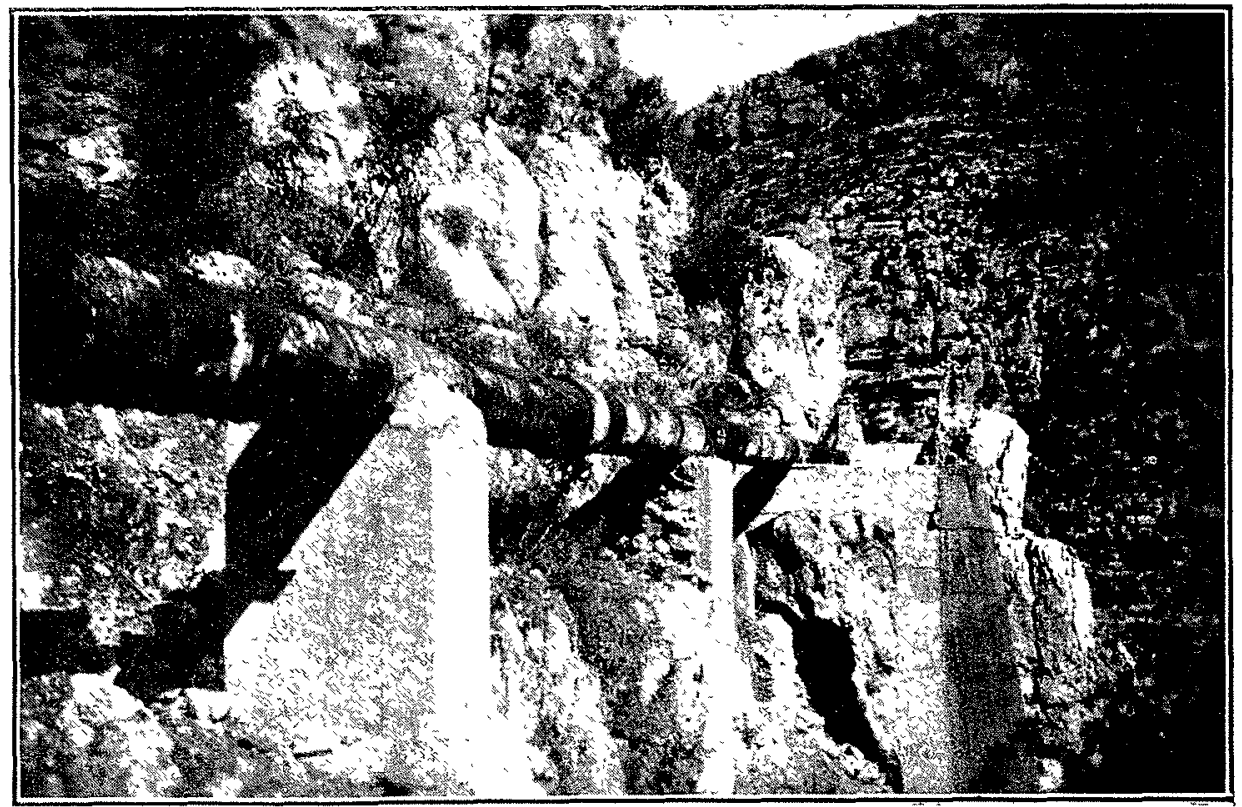

FIG. 5. - Tronçon diff jcile de la canalisation.

accumulée dans le bassin d'émergence. Le barrage en béton dont la fondation est enfoncée dans la couche imperméable du fonds du bassin est muni de deux déversoirs d'un mètre de longueur chacun, par lesquels l'eau arrive dans le réservoir de réception. Par un mur transversal de $35 \mathrm{~cm}$. d'épaisseur, ce

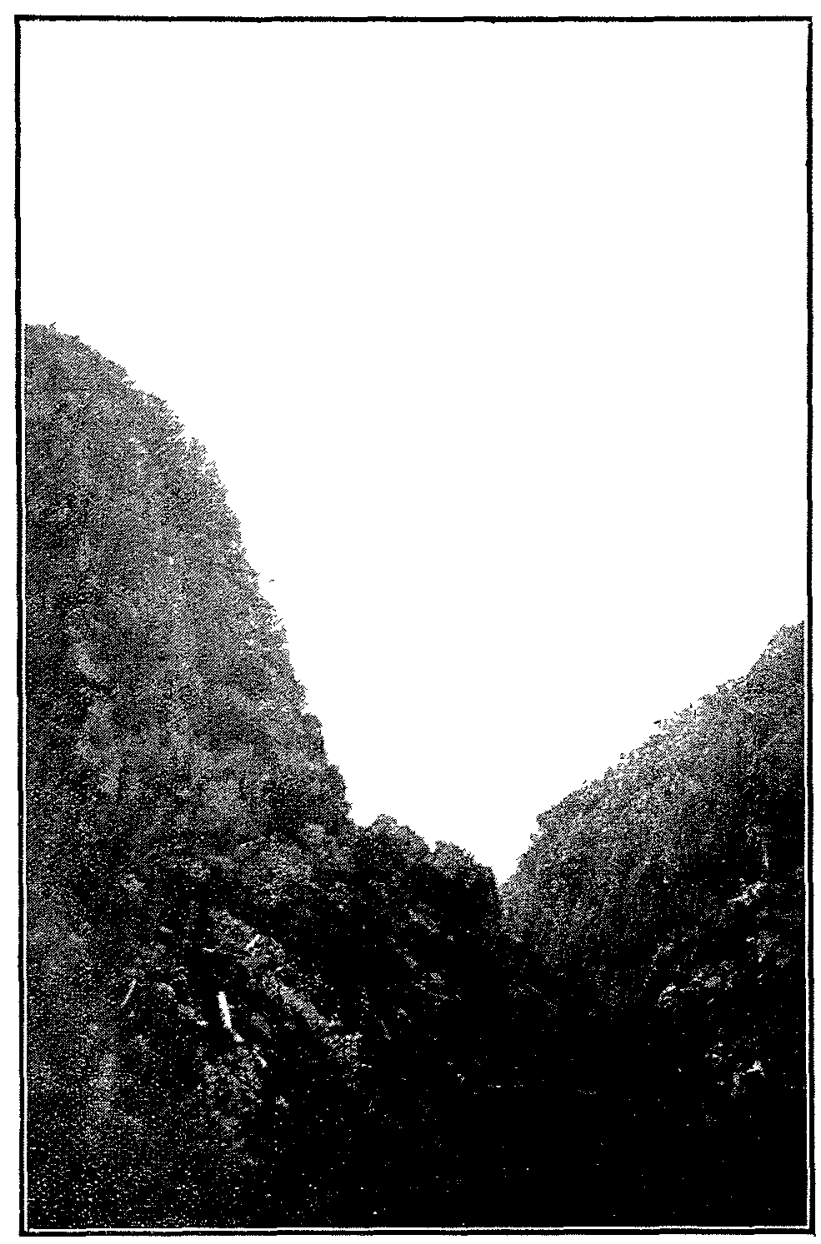

Fıg. 4. - Tronçon difficile de la canalisation. 
réservoir est partagé en deux compartiments indépendants où débouchent les tubes de la canalisation principale de $325 \mathrm{~mm}$.

Comme on l'a dit déjà, la canalisation principale, de $14 \mathrm{~km}$. de longueur, a pour but d'amener $10.000 \mathrm{~m}^{3}$ par jour d'eau de la source au réservoir de Jamhour dont le trop-plein $\epsilon$ st situé à la cote 512,55 . La différence d'altitude est donc de $126.30 \mathrm{~m}$.

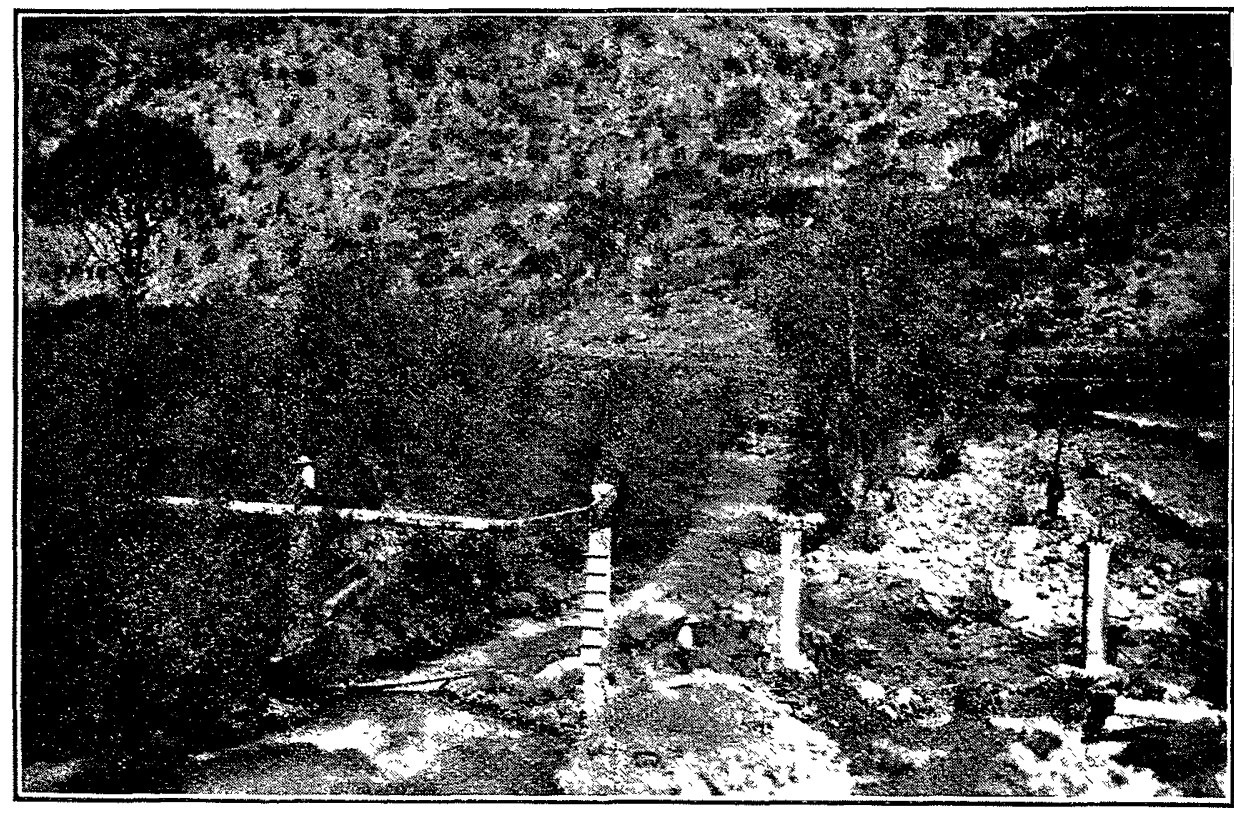

Frg. 6. - Aqueduc sur colonnes en ciment armé.

Le choix du tracé de cette conduitea présenté des difficultés assez sérieuses. Les versants de la vallée où passe la canalisation principale sont rocheux par excellence et souvent difficiles pour le passage, mème à pied Ils sont coupés par de nombreux talwegs et ravins. Surtout les tronçons entre les kilomètres 3 et 4 et entre les kilomètres 5 et 8 (massif d'Abadieh) où ce Lracé longe les flancs des montagnes (rocheux el abrupts) ont été pénibles, d'abord pour les études et ensuite pour l'exécution des travaux de construction. Ces tronçons, difficilement accessibles, ont demandé de nombreux ouvrages d'art afin d'assurer le passage de la canalisation.

Les figures 3,4 et 5 donnent une idée du terrain de ces troncons.

La pose de la canalisation principale a exigé environ 450 coudes 45 passages sur des colonnes supports en maçonnerie et en béton armé (fig. 6) pour les traversées des ravins, 3 passerelles en béton armé pour les traversées de la rivière (lig. 7) et 17 ventouses. Le tracé de la canalisation principale a été choisi en tenant compte de ce que la pression dans les tubes, au débit de $10.000 \mathrm{~m}^{3} \mathrm{j}$. ne dépasse pas, pour les joints normaux, 9 atmosphères. Là où on a été obligé d'accepter une pression dépassant quelque peu cette limite, les tubes aux joints rigides ont été employés.

Dans la ligne secondaire, donc après le grand réservoir de Jamhour, il y a des points où la pression dépasse 20 atmosphères. C'est là qu'ont été constroncons. truils des brises-charge en naçonnerie avec le loit et le sol en ciment armé. La pression maximum sans brise-charge s'élèverait à 20 atm.; en élablissant un brisecharge, on la réduil à $10 \mathrm{~atm}$., pression sans excès pour des tubes en acier. Il faul que les canalisations d'arrivece el celles de départ ne se trouvent pas en ligne droile pour qu'elles ne fonctionnent pas comme injecteur en entrainanl de l'air. Nous avons supprimé cet inconvénient en les appliquant l'une à gauche el l'autre à droite.

La fig. 8 présente en schéma le profil en long de la canalisation principale. Il est évident que dans des conditions pareille's, sans route d'accès à la canalisation projetér (la chaussée se trouve à une dislance de 500 à 3.000 mètres) on ne pouvait choisir que les tubes en acier. On a employé des tubıs sans soudure avec joints normaux ou rigides pour emboitement au plomb, asphaltés julés et chaulés. L'asphaltage et le jutage servent à protéger les tubes contre la rouille el aussi contre la terre agressive. La région où passe la canalisation n'a pas de lerre agressive, mais on a pris le plus sûr; des marécages peuvent se former quelque part et l'acide humique attaq uer les tubes. Contre ces mallvaises influences, on a prévu un asphallage et jutage, et, enfin, contre la chaleur, les tubes ont été chaulés. Cette dernière précaution ne coûte rien et vaut beaucoup, surtout pour les canalisations qui ne sont pas recouvertes de terre.

Les tubes ont été livrés par la S. A. d'Escaut el Meuse, dont nous désirons faire l'éloge pour l'excellente qualité des tubes et la promptitude des délais de livraison. Les longueurs varienl de 5,5 à 10,5 mètres. Les tuyaux courts de $5 \mathrm{~m}$. en moyenne ont été utilisés pour les tronçons à accès difficile dans les défilés rocheux, les tuyaux longs, de $8 \mathrm{~m}$. en moyenne, pour les autres

Leur transport de la chaussée jusqu'à la ligne des tranchées s'effectua à iorce d'hommes, ce qui présenta de grandes difficultés, mais qui coûta beaucoup moins que louteautre móthode

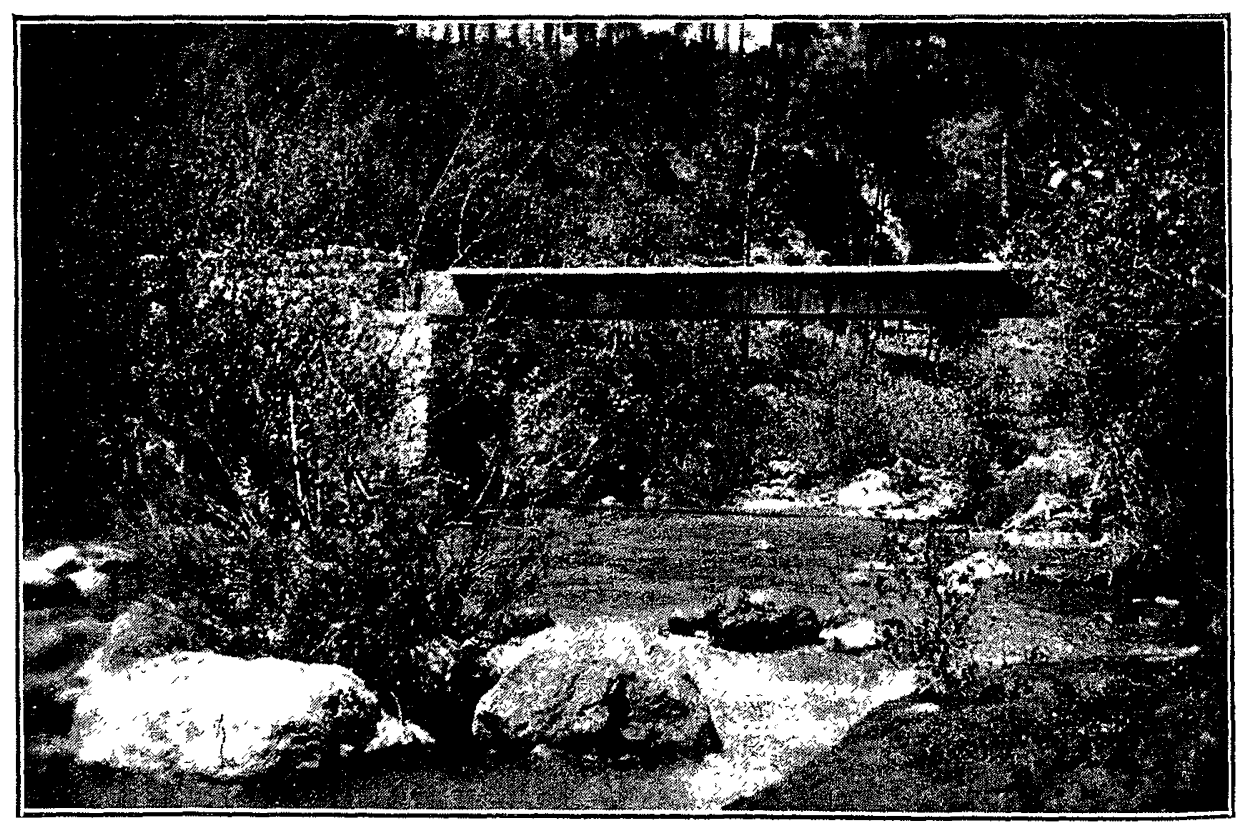

Fig. 7. - Aqueduc en béton armé. 
de transport (p. ex. celui à l'aide des transporteurs aériens). Là où c'élait possible, les tubes ont été transportés par des charriots. Pour le transport d'un tube de $8 \mathrm{~m}$. de longueur (poids $500 \mathrm{~kg}$. env.), 16 à 20 hommes ont été nécessaires. Malgré toutes les difficullés, le transport s'est effectué sans aucun accident sérieux. Quelques tubes seulement eurent le bout tordu. La

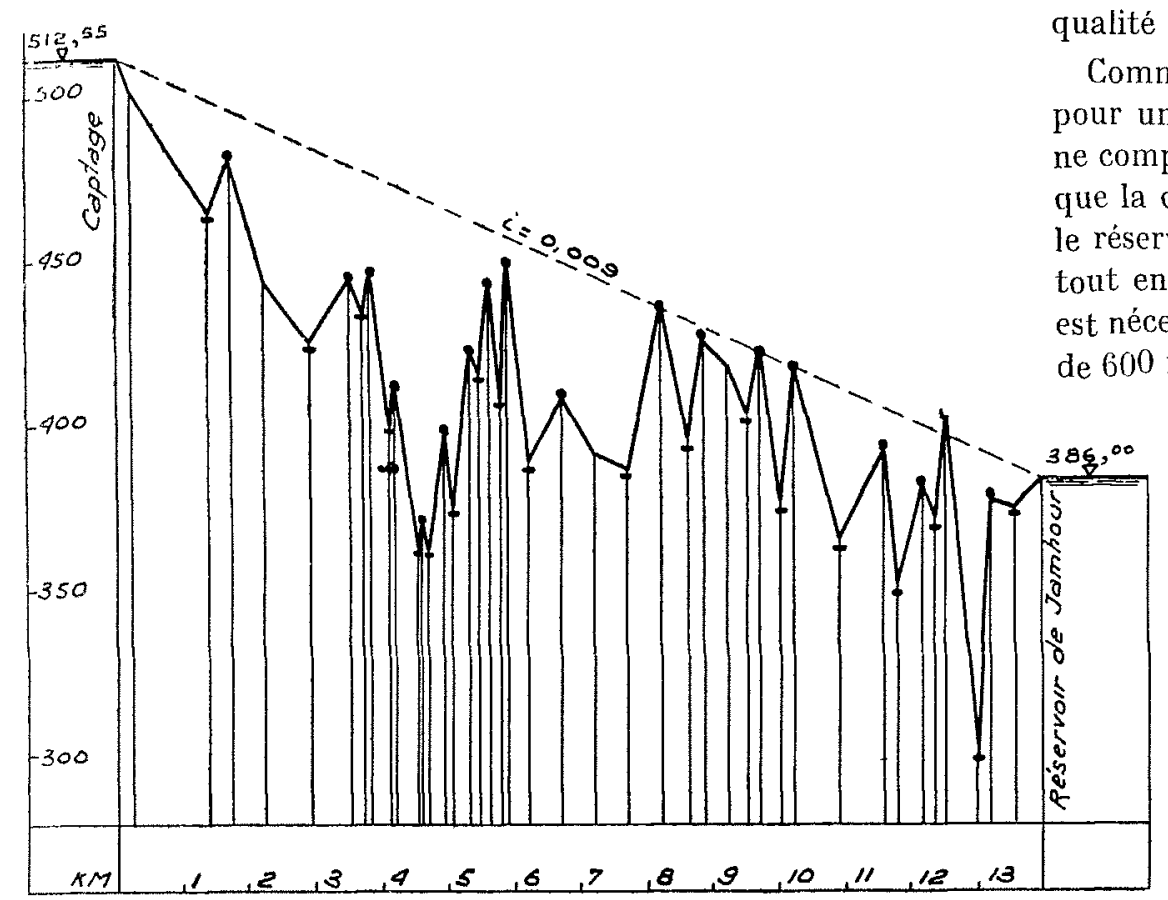

lili 8. - Canalisation principale Ain-el-DelbehJambour Profil réduit. Echelle horizontale 1.100.000 Echelle verticale 1. 2.000

couche de jute a beaucoup souffert de la manutention, de sorte qu'une équipe spéciale de 4 ouvriers s'occupait de la réparation avant que les tubes fussent fosés dans la tranchée.

Etant donné l'importance de la canalisation et les pressions considérables régnant par ci par là dans les tubes, le matage des joints a été confié aux mateurs spécialistes hollandais de la Maison Visser et Smit, Papendrecht (Hollande), entrepreneurs et actionnaires de la Société Générale d'Entreprises Hydrau- liques. L'équipe de matage comprenait un chef d'equipe, 2 mateurs (hollandais), 2 aides engagés sur place et 4 ouvriers. L'équipe de pose des tubes comprenait un chef et 12 à 16 ouvriers. La pose et le matage des $14 \mathrm{~km}$. de la canalisation principale ont été effectués en 5 mois (fin décemb e-fin mai). Les épreuves des joints par la pression, allant jusqu'à 15 atm. et même jusqu'à $22 \mathrm{~atm}$. dans la vallée de Jamhour, ont démontré la haute qualité du travail des mateurs hollandais.

Comme déjà mentionné plus haut, la canalisation est calculée pour un débit max. de $116 \mathrm{l} / \mathrm{s}$. $\left(10.000 \mathrm{~m}^{3} / \mathrm{j}\right.$.), mais la Société ne compte pas, pendant les premières années de fonctionnement, que la consommation dépasse $4.000 \mathrm{~m}^{3}$ par jour. C'est pourquoi le réservoir de Jamhour est construit seulement pour $1.200 \mathrm{~m}^{3}$, tout en prévoyant que sa capacité pourra être doublée, si cela est nécessaire. Le réservoir en béton armé a deux compartiments de $600 \mathrm{~m}^{3}$ chacun. La vue générale en est présentée sur la fig. 9 .

Pour les canalisations secondaires d'adduction, on utilisa également les tubes en acier, les. diamètres variant de 100 à $225 \mathrm{~mm}$. Les bases de leurs projets sont les mêmes que pour la canalisation principale. Mais, évidemment, les conditions de construction furent plus faciles, étant clonné la région où passent ces canalisations, mieux 'esservie par les voies de communication le terrain plus accessible et le poids considérablement inférieur. Quant aux réservoirs locaux, celui de Hazmieh $\left(350 \mathrm{~m}^{3}\right)$ est du même type que le réservoir de Jamhour et les deux autres (Sinn et Fil) $350 \mathrm{~m}^{3}$ et (Baabda) $100 \mathrm{~m}^{3}$ ont leurs murs de pourtour en maçonnerie et le sol en béton.

Les travaux ont été exécutés par la $\mathrm{S}$. G. d'Entreprises? Hydrauliques en régie. Les études et la rédaction du projet de la canalisation d'adduction ont été confiés à Mr. B. Litchkousse, ingénieur en chef de la Société. La révision du projet en vue de l'exécution, avec la confection des projets et dessins des réservoirs a été confiée au soussigné. La surveillance des travaux a été partagée entre les agents de la Société et la Maison Visser et Smit, représentée par M. Abraham Visser, Directeur, et l'Administrateur de la Société, M. Henri Helou, Beyrouth.

Avec la construction de cette adduction, un beau travail a été réalisé dans un beau pays.

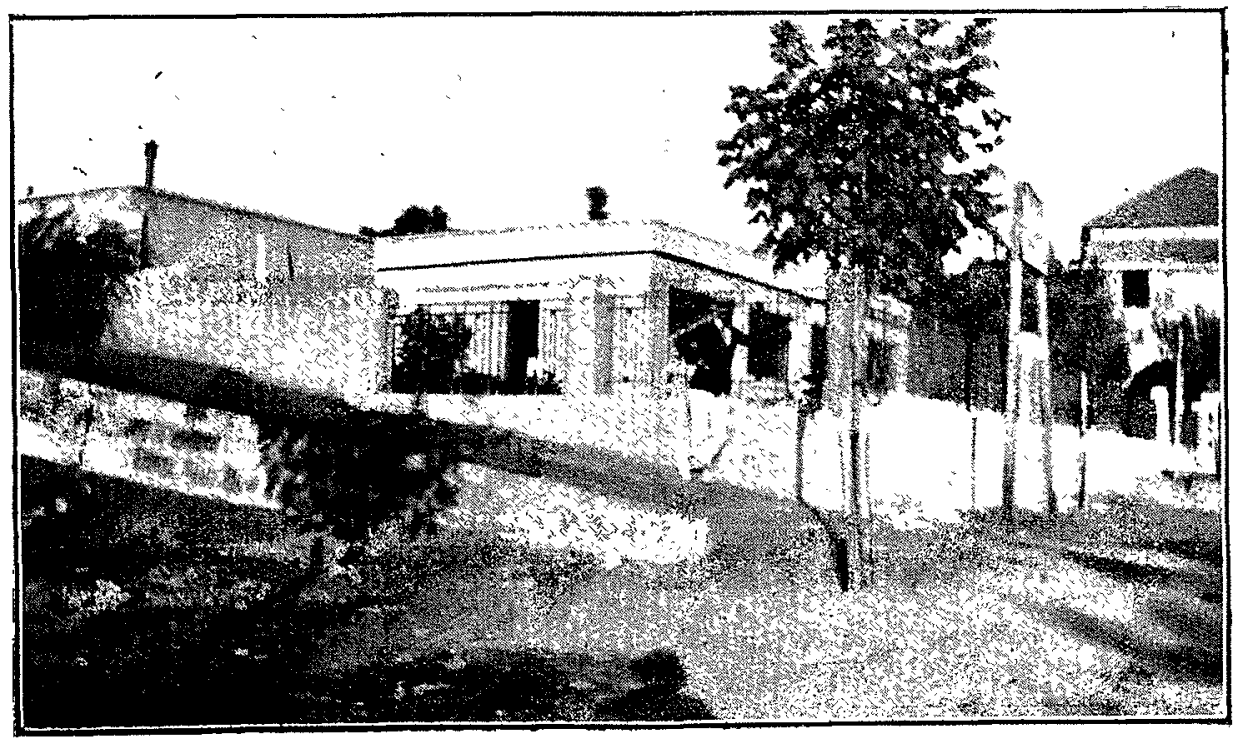

Fłg. 9. - Grand réservoir de Jambour 